\title{
MAYER-ROKITANSKY-KUSTER-HAUSER SYNDROME (MRKHS), A RARE CAUSE OF PRIMARY AMENORRHOEA: THREE CASE REPOR'TS
}

\author{
Karuppiah D $\mathbf{D}^{1}$, Dilakkumar $\mathrm{S}^{2}$. \\ ${ }^{1}$ Consultant Endocrinologist, Teaching Hospital Batticaloa, ${ }^{2}$ Consultant Radiologist, Base Hospital Kalmunai.
}

\section{ABSTRACT}

Primary amenorrhoea is one of the common reproductive disorders affecting females and it could be due to may causes including structural, functional and endocrine abnormalities. We reported three young women presented with primary amenorrhoea, where the investigations confirmed the diagnosis of Mayer-Rokitansky-Kuster-Hauser Syndrome (MRKHS) in all three cases. Our objective of reporting these case series was to highlight the importance of careful evaluation the of all patients with primary amenorrhoea and to avoid unnecessary oestrogen treatment.

Running title: Amenorrhoea due to MRKHS

Keywords: Primary amenorrhoea, MRKH syndrome, Genitourinary anomalies.

\section{INTRODUCTION}

Primary amenorrhea is defined as the failure of the initiation of the normal menstruation by age 16 years (1). During normal puberty, the endocrine axis comprising the hypothalamus, pituitary, and ovaries as well as an intact uterus and patent vaginal tract will ensure menarche and regular normal menstrual flow. Disorders affecting the hypothalamus, pituitary gland and ovaries, chromosomal anomalies and genitourinary malformations can lead to disturbance to normal pubertal process causing primary amenorthoea. Congenital abnormalities affecting female reproductive tract are responsible for about one-fifth of the patients with primary amenorrhoea (2). Mayer-Rokitansky-Kuster-Hauser syndrome (MRKHS) is a disorder that leads to structural abnormalities in the uterus and vagina due to agenesis or partial agenesis of the Mullerian duct system. We describe 3 patients who were presented with primary amenorrhoea and found to have MRKH syndrome.

CASE 1:

A 20-year-old girl who was planning for marriage in near future presented with a history of primary amenorrhoea. She has no previous medical history or cyclical abdominal pain. Her family history was unremarkable. Her height was $155 \mathrm{~cm}$ and weight was $50 \mathrm{Kg}$. Breast development was at Tanner Stage $V$, and pubic hair was Tanner stage III and had normal axillary hair. There was no clinical feature suggestive of Turner syndrome or other dysmorphic features. Rest of the examination was normal.

Her biochemical and endocrine evaluation revealed normal results (Table 1). She had 46XX karyotype without numerical or structural abnormalities. Trans-rectal ultrasound scan demonstrated absent uterus with a short blind pouch of the vagina and normal size ovaries (figure 1). There were no renal anomalies.

\section{CASE 2:}

The second patient was a 19-year-old girl who presented with primary amen-orrhoea. She did not give a history of cyclical abdominal pain. She was treated with combined oestrogen pills (OCP) for few months. However, the OCP failed to induce her periods. She has no significant medical or family history. Her mother and elder sister attained menarche at the age of 14 years. She was $158 \mathrm{~cm}$ in height and $54.9 \mathrm{Kg}$ in weight. She had normal secondary sexual characteristics with breast development and pubic hair at Tanner Stage $\mathrm{V}$ with normal axillary hair. Systemic examination was normal and she had no features of Turner syndrome.

Her biochemical and hormone profile was shown in table 1 . She had normal Karyotype with 46XX. The ultrasound scan showed normal renal system, but the trans-rectal scan showed absent uterus with normal size ovaries (Figure 2).

\section{CASE 3:}

The third patient is a 15 -year-old girl who also referred to our endocrine unit with primary amenorrhoea. Her medical history was unremarkable and she was active and good at her studies. Apart from maternal diabetes, she has no significant family history. There was no history of consanguinity and there were no other family members with menstrual problems. Her height was $154 \mathrm{~cm}$, weight was $48 \mathrm{Kg}$, and blood pressure was $120 / 70 \mathrm{mmHg}$. Breast development was at Tanner stage IV, and pubic and axillary hair 


\section{Table 1: Biochemical parameters of the patients}

\begin{tabular}{lrrrr}
\hline Test & Case $\mathbf{1}$ & Case 2 & Case 3 & Normal range \\
\hline Biochemical tests & & & & \\
Haemoglobin & 11.8 & 13 & 13.2 & $12-15 \mathrm{~g} / \mathrm{dl}$ \\
Serum Creatinine & 0.8 & 0.7 & 0.73 & $0.7-1.3 \mathrm{mg} / \mathrm{dl}$ \\
Sodium & 138 & 142 & 140 & $135-145 \mathrm{mmol} / 1$ \\
Potassium & 4 & 3.7 & 3.9 & $3.5-5 \mathrm{mmol} / 1$ \\
Calcium & 2.31 & 2.28 & 2.20 & $2-2.26 \mathrm{mmol} / 1$ \\
Fasting plasma glucose & 89 & 97 & 78 & $70-100 \mathrm{mg} / \mathrm{dl}$ \\
ALT & 17 & 8 & 12 & $5-530 \mathrm{U} / 1$
\end{tabular}

\begin{tabular}{|c|c|c|c|c|}
\hline \multicolumn{5}{|l|}{ Hormone tests } \\
\hline Oestradiol & 258.8 & 111.9 & 234.12 & $21-313(\mathrm{pg} / \mathrm{ml})$ \\
\hline Testosterone & 0.2 & 0.8 & 1.16 & $0.52-1.4(\mathrm{nmol} / \mathrm{l}))$ \\
\hline Follicular stimulating hormone & 5.91 & 4.66 & 1.05 & $1.38-9.58(\mathrm{IU} / \mathrm{l})$ \\
\hline Luteinizing hormone & 6.2 & 2.3 & 0.81 & $0.8-15(\mathrm{IU} / \mathrm{l})$ \\
\hline Serum prolactin & 466 & 245 & 321 & $78-490(\mathrm{mIU} / \mathrm{l})$ \\
\hline Thyroid stimulating hormone & 3.7 & 3 & 2.39 & $0.5-5(\mathrm{mIU} / \mathrm{L})$ \\
\hline Free $\mathbf{T} 4$ & 0.98 & 1.2 & 1.02 & $0.8-2(\mathrm{ng} / \mathrm{dl})$ \\
\hline
\end{tabular}

was at Tanner stage III. Examination of female external genitalia was normal. There was no evidence of Turner's syndrome or facial dysmorphism, webbing of the neck, or scoliosis.

Laboratory investigations and endocrine evaluation were normal

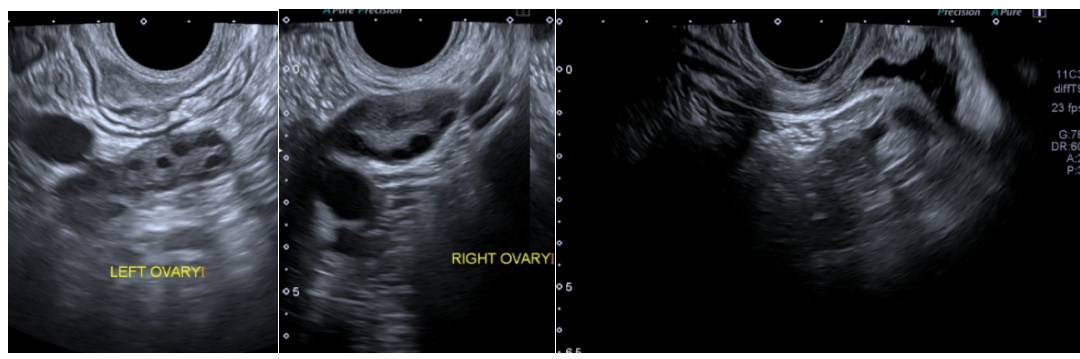

Figure 1. Trans-rectal ultrasound scan shows normal ovaries and absent uterus in case 1.

(Table 1). The Karyotype was $46 \mathrm{XX}$, and no $\mathrm{Y}$ chromosome material was detected. The ultra sound scan showed normal size ovaries and the visualization of the uterus was difficult.
MRI scan of pelvis revealed very small rudimentary uterus and normal size ovaries with immature follicles bilaterally. She has no renal abnormalities. Cardiac evaluations including 2D Echo, auditory examinations, and the bone studies were normal in these three subjects.

of the female secondary sexual characteristics and functioning ovaries with normal 46XX karyotype (3). Approximately 1 in 5000 female births is later diagnosed with MRKHS (3).

MRKHS is of two types. The isolated absence of the proximal two-thirds of the vagina is the only abnormality that is seen in MEKHS type 1, whereas in type 2, other malformations, such as vertebral, cardiac, urologic (upper tract), and otologic anomalies are also seen in addition to the vaginal abnormalities. However, their ovarian function is normal. Therefore, these patients go through the puberty with normal thelarche and adrenarche and they have normal external genitalia. Due to upper vaginal and uterine problems, they will not have menstrual periods and primary amenorrhoea and infertility are the main clinical presentations. Depending on the degree of vaginal aplasia, the patients can also experience dyspareunia during vaginal intercourse. Patients with a uterus, 
but without an outflow pathway may report cyclical abdominal pain due to endometrial shedding. Patients with type 2 MRKHS usually have vertebral anomalies, urinary incontinence, and can also have voiding difficulties due to associated skeletal and renal anomalies (3). According to the clinical finding, all three patients in our study had features suggestive of type 1 MRKHS.

Although MRKHS was thought to be a sporadic anomaly, increasing number of familial cases suggest the possibility of a genetic aetiology (2). This syndrome appears to be transmitted in an autosomal dominant fashion, with incomplete penetrance (4). Abnormal activation of anti-mullerian hormone or receptor and mutations in WNT4 were also hypothesised. However, the main factor responsible for this abnormal development was not proven by any of these hypotheses $(5,6)$.

These patients have the normal ovarian function with normal circulating levels of $\mathrm{LH}$ and FSH. When there is a suspicion of Androgen insensitivity syndrome (AIS), testosterone analysis is warranted. The levels will be in the normal female range in MRKH syndrome and high levels (normal male range) are seen in AIS. Chromosomal analysis is an essential investigation to exclude karyotype abnormalities such as Turner syndrome and AIS, two other main causes of primary amenorrhoea (7). Our patients had normal 46XX karyotyping and normal ovarian function with testosterone levels with-in the normal female range.

Urogenital imaging is an essential component of their clinical evaluation. Ultrasonography of pelvis and perineum can provide an excellent view of the upper level of the vagina and the length of its obstruction and it helps to identify uterine anomalies and tubal obstructions. It also provides an assessment of kidneys and bladder at the same time. Conventional transabdominal, transrectal or trandlabial sonography can be considered for this purpose. Magnetic resonance imaging (MRI) is more sensitive in detecting active endometrial tissue and it is helpful in

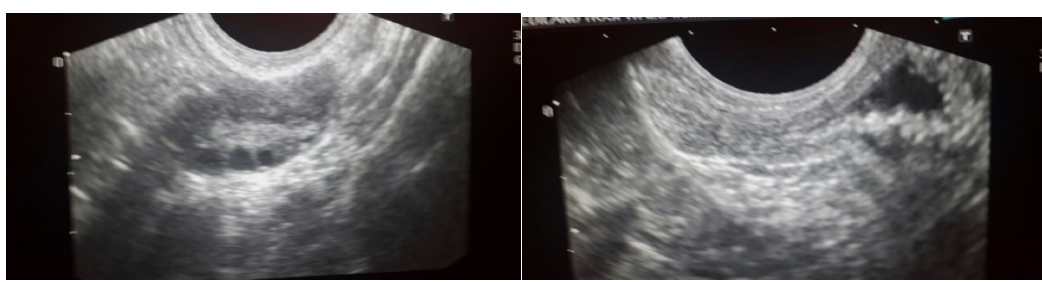

Figure 2. Trans-rectal ultrasound scan showing normal size ovaries and absent uterus in case 2

patients with inconclusive ultrasonography findings concerning uterine cavity (8). MRI can also provide additional information regarding vertebral anomalies and MR urography can be used to assess urinary tract in type $2 \mathrm{MRKH}$ syndrome when urinary tract abnormalities are suspected. However, intravenous pyelography may be needed to assess renal structure in some patients (3). Laparoscopy is another diagnostic and therapeutic procedure that is recommended for patients who are experiencing cyclical pain to remove uterine remnants and endometriosis.

Management of patients with MRKHS should address functional as well as the emotional aspect of reproductive tract anomalies. Primary amenorrhoea, infertility, and sexual intercourse difficulties are the major concerns. Other structural anomalies involving renal, auditory, cardiac and skeletal system are added problems that need to be addressed during the management of patients with type $2 \mathrm{MRKH}$ syndrome. Diagnosis of MRKHS itself impairs the quality of life of these patients by limiting their expectations and individual life goals. This is mainly through to be due to the psychological distress related to the impossibility to carry a pregnancy and the social pressure related to that $(9,10)$. Once the diagnosis of MRKH syndrome is made, it is, therefore, important that the young girls are provided with adequate psychological counselling with special emphasis on disease-related sexual problems.

The main focus of MRKHS treatment involves creating a neovagina to fascilitate normal vaginal intercourse. Various methods ranging from the non-surgical approach to surgical procedures have been described for creating a new vagina (11). Nonsurgical options such as selfadministered perennial dilatation can be tried on well-motivated patients. However, depending on the degree of vaginal defect, the majority will end up having surgical interventions. Although there is no consensus regarding surgical approach, skin grafts, vulval flaps, various fasciocutaneous flaps and flaps from an isolated segment of bowel are used to create a functional vagina in these patients (12-14). Patients who experience cyclical pain related to endometriosis may require surgical excision of the uterine remnants and the rudimentary uterus (15).

Achieving normal fertility is a major challenge for these patients. Although familial aggregates of MRKHS have been documented, the female offsprings of affected females, who got conceived by assisted reproductive techniques, usually have normal reproductive tracts (16). Therefore, discussion regarding assisted reproductive techniques and the use of a surrogate mother is also appropriate in relevant patients. Helping them to understand their reproductive potential to become a biological parent may help them to deal with this complex diagnosis in a favourable manner.

Vaginal dilatation and surgical repair in an unmarried girl have added cultural impacts in our clinical setup. None of our patients were willing to undergo these procedures straightaway. All the three patients and their parents required counselling in order to address their concerns and emotional feelings. The imaging of the patient had cyclical pain revealed 
rudimentary uterus and she was advised to report back for reevaluation for the development of endometriosis. The uterus was absent in other two patients.

\section{CONCLUSION}

Proper diagnosis and comprehensive evaluation for associated anomalies are essential in managing patients with MRKHS. Psychological counselling is vital to address the functional and emotional effects of reproductive tract anomalies. Although non-surgical vaginal dilatation is considered as first line approach, the majority will need surgical interventions. However, surgical reconstruction has not been able to establish the ability to conceive through natural means. Because of the normally functioning ovaries, a conception of a genetic offspring is possible with the aid of assisted reproductive techniques through the use of surrogate mother. However, the emotional impact related to surrogacy and the added legal issues related to this process can be frustrating for the patients and the family.

\section{REFERRENCES}

1. Kenneth M Bielak. Amenorrhea. Medscape updated: Mar 02, 2016.

2. Sahbi Kebaili, Kais Chaabane, Mouna Feki Mnif, Mahdi Kamoun, Paten Hadj Kacem, Nouha Guesmi et al. Gonadal dysgenesis and the Mayer-RokitanskyKuster-Hauser Syndrome in a girl with a 46, XX karyotype: A case report and review of the literature. Indian Journal of Endocrinology and Metabolism 2013; 17(3): 505-508.

3. Andrew J Kirsch. Mayer-RokitanskyKuster-Hauser syndrome - practice essentials. Medscape updated: Apr 08, 2016.

4. Morcel K, Guerrier D, Wartin T. The MRKH syndrome: clinical description and genetics. Journal of Gynaecology, obstetrics, Biology and Reproduction (Paris) 2008; 37(6): 539-546.

5. Oppelt P, Strissel PL, Kellermann A, Seeber S, Humeny A, Beckmann MW, et al. DNA sequence variations of the entire anti-Mullerian hormone (AMH) gene promoter and AMH protein expression in patients with the MayerRokitanski-Kuster-Hauser syndrome. Human Reproduction 2005; 20: 149-157.
6. Philibert P, Biason-Lauber A, Rouzier R, Pienkowski C, Paris F, Konrad D, et al. Identification and functional analysis of a new WNT4 gene mutation among 28 adolescent girls with primary amenorrhea and müllerian duct abnormalities: A French collaborative study. Journal of Clinical Endocrinology and Metabolism 2008; 93: 895-900.

7. Londra L, Chuong FS, Kolp L. MayerRokitansky-Kuster-Hauser syndrome: a review. International Journal of Women's Health 2015; 7: 865-870.

8. Torres-de la Roche LA, Devassy R, Gopalakrishnan S, De Wilde MS, Herrmann A, Larbig A, et al. Plastic neovaginal construction in MayerRokitansky-Küster-Hauser syndrome: an expert opinion paper on the decisionmaking treatment process. GMS Interdisciplinary Plastic and Reconstructive Surgery DGPW 2016; 5: Doc08.

9. Laggari V, Diareme $S$, Christogiorgos $S$, Deligeoroglou E, Christopoulos P, Tsiantis J. and Creatsas, G. Anxiety and Depression in Adolescents with Polycystic Ovary Syndrome and MayerRokitansky-Kuster-Hauser Syndrome. Journal of Psychosomatic Obstetrics and Gynecology 2009; 30: 83-88.

10. Krupp K, Fliegner M, Brunner F, Brucker S, Rall K, Richter-Appelt H. Quality of life and psychological distress in women with Mayer-RokitanskyKüster-Hauser-Syndrome and individuals with complete androgen insensitivity syndrome. Open Journal of Medical Psychology. 2014; 3: 212-221.

11. Deans R, Berra M, Creighton SM. Management of Vaginal Hypoplasia in Disorders of Sexual Development: Surgical and Non-Surgical Options. Sexual Development. 2010; 4: 292-299.

12. Bianchi S, Frontino G, Ciappina N, Restelli E, Fedele L. Creation of a neovagina in Rokitansky syndrome: comparison between two laparoscopic techniques. Fertility Sterility. 2011; 95(3): 1098-1100.

13. Roberts CP, Haber MJ, Rock JA. Vaginal creation for müllerian agenesis. American Journal of Obstetrics and Gynaecology 2001; 185(6): 1349-1352.

14. Veronikis DK, McClure GB, Nichols DH. The Vecchietti operation for constructing a neovagina: indications, instrumentation, and techniques. Obstetrics \& Gynaecology 1997; 90(2): 301304.

15. Committee opinion: no. 562: müllerian agenesis: diagnosis, management, and treatment. Obstetrics \& Gynecololgy 2013; 121(5): 1134-1137.

16. Petrozza JC, Gray MR, Davis AJ, Reindollar RH. Congenital absence of the uterus and vagina is not commonly transmitted as a dominant genetic trait: outcome of surrogate pregnancies. Fertility and Sterility. 1997; 67: 387-9. 